\title{
Why mothers choose to enrol their children in malaria clinical studies and the involvement of relatives in decision making: Evidence from Malawi
}

\section{Francis Masiye ${ }^{1}$, Nancy Kass ${ }^{2}$, Adnan Hyder ${ }^{2}$, Paul Ndebele ${ }^{1}, J_{0 s e p h}$ Mfutso-Bengo ${ }^{1}$}

1. Centre for Bioethics in Eastern and Southern Africa, CEBESA, College of Medicine, Malawi.

2. Department of Health Policy and Management, Johns Hopkins Bloomberg School of Public Health, Baltimore, Maryland, USA.

Corresponding author: Francis Masiye, Centre for Bioethics in Eastern and Southern Africa (CEBESA), Department of Community Health, College of Medicine, Private Bag 360, Chichiri, Blantyre 3, Malawi Phone: +265 1871 911, Email:fmasiye@medcol.mw

\section{Abstract}

This study was aimed at researching the reasons why mothers enrol their children in malaria clinical research and how family members or relatives are involved in the decision-making process. Issues related to informed consent were also a particular focus of this study. A total of 81 participants took part in 8 focus group discussions. Thirty-nine participants were recruited from Blantyre, an urban setting, and fortytwo participants were from Chikwawa, a rural setting. All the participants were mothers whose children had participated or were participating in the Intermittent Prevention Therapy post-discharge (IPTpd) Malaria Research.A majority of the participants reported that they chose to participate in the IPTpd research as a way of accessing better quality medical care. They also decided to enrol their children in order to benefit from the material and monetary incentives that were being given to participants for their participation. Most participants reported that they made the enrollment decisions on their own. They informed their family members/marital partners about their enrollment decisions after they had given their consent. A few made their enrollment decisions after consulting their family members. There was also a sense of trust in health workers who asked the potential participants to join the IPTpd research. Most participants decide to take part in malaria research because of better medical treatment. Partners and relatives play a very small role in the decisionmaking process of participants in malaria clinical research. Research participants have a sense of trust in health workers who enrol them in clinical research.

\section{Introduction}

For the past decade, researchers have noted that obtaining individual informed-voluntary consent is generally difficult in some social-cultural contexts, especially in poor-resource settings. Although the principle of informed consent is universal, the implementation of its procedures may be different in developing countries, requiring that they be tailored to local custom and culture ${ }^{1}$. Three studies conducted with rural populations highlight the practice of involving family members and the difficulties in obtaining informed and voluntary consent.

One such study conducted in rural Peru reported that the consent to participate in research is most commonly a family decision and that fathers and grandmothers are frequently influential in the decision-making process ${ }^{2}$. In that setting, individual potential participant do not decide independently whether to enrol in research. The decision whether to enrol in a study or not often depends on the family members.

And in order to ensure that potential participants and their family members make an informed decision, researchers who conduct research in such settings make an effort to inform both potential participants and their families about the research before potential participants give their consent to participate. However, the study does not state clearly how family members' decisions influence the voluntariness of individual participants' decisions.

A similar study was conducted in rural Ghana ${ }^{3}$. In their study, it was reported that female participants who are asked to enrol in research generally consult their husbands before they decide to participate and that their husbands' wishes affect their decisions of whether to participate or not. The researchers also reported that community leaders permit research to be conducted in their communities based on their trust of researchers and that individuals enrol in research based on their trust of community leaders. Although husbands are decision-makers in such settings, the study does not say whether both female participants and their partners are fully informed about the research. The study also does not say how husbands' decisions affect the voluntariness of potential participants' consent to participate in research.

The above sentiments on husbands making decisions for their wives are also articulated in a study conducted in rural Uganda by Nelson Sewankambo. Sewankambo has noted that in rural areas, the family head, who is normally a husband, is widely recognized and he is expected to take final decisions in all matters affecting family members. In such areas, family members who do not submit to such decisions may face serious consequences including domestic violence and/or divorce. Thus, in such circumstances women and children will tend not to participate in a study unless permission has been granted by the head of the household. ${ }^{4}$ Taking Sewankambo's finding into consideration, the Ugandan National Research Council has included a clause in its ethical guidelines for biomedical research that urges researchers to obtain permission from household heads before they

involve a member into their studies Similar research was also conducted with urban populations. In a study conducted by Christine Pace and others in peri-urban Uganda, it was reported that $94 \%$ of the 347 participants personally made the decision to enrol their children in malaria studies while $6 \%$ indicated that they sought help from their spouses or family and friends before deciding to take part in the study ${ }^{6}$.

In a paper written by Anant Bhan and others, it was reported that most individuals in urban India do not agree to participate in research or even to major clinical procedures without discussing it with one or more members of their families? ${ }^{7}$. It was also reported that women do not feel empowered enough to agree to participate in research until they obtain the permission of their spouses. In the same paper, Adejumo, a Nigerian, states that Nigeria is " $a$ male dominated society and this being the case, women often need to obtain their spouse's permission before participating in research"' 
He also states that before recruiting participants from any community, community heads, local chiefs, compound heads and family heads must be consulted. Mina, an Iranian, also reports that Iranians have respect for the family as a social unit and family members consult and support each other. She goes on to say that "when a spouse participates in a nontherapeutic research in which the consequences might affect marital life,

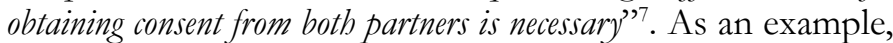
she says in order to perform either a vasectomy or a tubal ligation, it is necessary to obtain written informed consent from the husband and wife.

From the above studies, it is evident that family members are involved to varying degrees in the decision-making process in both urban and rural regions of some developing countries. Yet it is not clear whether their involvement affects the voluntariness of the decisions made by individual participants. It is also worthy to note that in some of the settings, the informed consent process involves family members, community leaders and the potential participants themselves. Based on this information, some researchers such as Caballero and Strauss have suggested family/ community involvement in the informed consent process 8 . However, other researchers feel that involving family/ community members might affect the voluntariness of the individual subject. For example, Hebert and DeCosta feel that family involvement may undermine individual autonomy and the whole process of individual informed consent ${ }^{9}$. The US National Bioethics Advisory Commission (NBAC) also acknowledges that there are difficulties in obtaining voluntary consent from subjects in some social and cultural contexts. In one of its recommendations, the NBAC urges researchers to take appropriate steps to accommodate the wishes of potential participants who would like to involve family members in the consent process but it cautions them not to let family involvement override voluntary consent ${ }^{10}$.

Evidently, the debate is going on. There are still unanswered questions on the complexity of obtaining individual informed consent and voluntary consent from subjects within their cultural, familial and social contexts. This is an ethical challenge facing researchers and sponsors of biomedical research in developing countries and Africa in particular. Surprisingly, very little has been done to examine this question empirically in Africa. Yet there is a growing number of research activities, raising questions of how research participants in an African social and cultural context make decisions about participation in research.

In the Malawian context, reports from various clinical studies indicate that many female potential research subjects ask for ample time to consult family members (marital partners) before deciding to participate in their clinical studies (Personal communication with Research Assistants from the Johns Hopkins Project in Blantyre and the UNC Project in Lilongwe). Perhaps family members (partners) significantly influence their decision to participate in clinical studies. In addition, findings from Phase II of the Wellcome Trust Bioethics Research Project indicate that family involvement in the decision-making process is common. Over $64 \%$ of the participants reported that they consulted their family members before making their decisions to participate in the clinical studies. ${ }^{11}$ This suggests that family members might influence the decision making process. However, none of the above studies sought to explore the differences in decision-making between urban and rural settings. They were either conducted in a peri-urban setting or an urban setting. Additionally, none of them involved people taking part in malaria clinical studies, malaria being one of the killer diseases in Malawi. Therefore, there is no empirical evidence on how research participants decide to enrol in malaria clinical studies and on the involvement of family members in the decision-making process or on the differences in decisionmaking between rural and urban participants in the same social-cultural context. None of the studies of which we are aware compared decision-making between urban and rural settings in the same context. Hence, the study attempted to fill this gap, specifically in the Malawian context. This was the first study to address this issue and it could contribute to the body of evidence on how research participation decisions in malaria clinical studies in both urban and rural settings are made, informing researchers in malaria clinical studies in Malawi on common differences in decision-making process between rural and urban settings.

\section{Methods \\ Study Design}

This was a descriptive interview study. The purpose was to explore factors which may influence participation of individuals in rural and urban clinical studies. The study employed qualitative research methods, specifically focus group discussions with female Malaria clinical study participants from rural and urban settings.

\section{Study Sites}

The study was conducted in two sites, an urban setting and a rural setting. The reason for conducting the study in both settings was to explore differences in the participants' decision making process between urban and rural settings. Participants in malaria clinical studies from a rural setting were recruited from Chikwawa District Hospital and those from an urban setting were enrolled at the Queen Elizabeth Central Hospital in Blantyre. The two sites were selected because they have been or are locations for several largescale clinical research projects. In addition, they are distinct from each other because one is rural and the other is urban. This distinction enabled us to examine rural and urban participants' reasons for enrolling in clinical research and how relatives and partners in both settings influence individual decisions to participate in clinical research.

\section{Study Population}

The target populations were supposed to be both male and female guardians of children who have participated (in the past one year) or are currently participating in the IPTpd (Intermittent Prevention Therapy post-discharge) Malaria clinical research. However, there were very few male guardians for participants in the IPTpd malaria clinical research. Therefore, we conducted focus group discussions with female guardians only. The participants were recruited in both rural and urban study sites. We recruited 81 participants for this study: 42 participants in the IPTpd Malaria clinical research from an urban setting (the Queen Elizabeth Hospital in Blantyre, Malawi); and 39 participants in the IPTpd Malaria clinical research from a rural setting (Chikwawa District Hospital, Malawi). The participants in each setting formed four focus groups of eight to twelve 
people each. In total, there were eight focus groups.

\section{Recruitment Procedures}

The IPTpd malaria research teams in the two sites assisted in identifying the eligible participants in the two sites. They approached female adult guardians (aged between 18 and 60) whose children participated (during the past year) or are currently participating in the IPTpd Malaria research and referred them to the Research Team. Members of the Research Team explained to each of the potential participants that a new study was being conducted that involved a focus group discussion to learn what motivated them to participate in the IPTpd clinical research. Those who expressed willingness to participate in the study were scheduled for a focus group discussion on a specific date.

All focus group discussions took place in a private place in both settings. Oral informed consent was obtained from each participant prior to participation. Each participant was assigned a unique identification code (for instance, P1 for participant 1). Individual responses were represented by the identification codes and the codes linked to the data generated from the focus groups.

In both settings, 81 participants were enrolled; 42 participants were recruited from the Queen Elizabeth Hospital and 39 participants from Chikwawa District Hospital.

\section{Ethical approval}

The study was classified as exempt from review by the Committee on Human Subjects Research (CHR study \# H.30.06.05.05 AX) at the Johns Hopkins Bloomberg School of Public Health in the US. It was approved by the College of Medicine Research and Ethics Committee (COMREC study \# P.10/07/589) in Malawi.

\section{Data Collection}

Data were obtained from research subjects during focus group discussions. The interview guide was translated into Chichewa and the focus groups were administered in Chichewa, the local language. All focus group discussions were audio taped. In the focus group discussions, participants were asked to explain how they decided to enrol their children in the IPTpd malaria research and the roles of their partners/relatives in the decision-making process. They were also asked to explain what motivated them to enrol their children in the IPTpd malaria research. These questions were asked of the participants as a group and each participant was encouraged to answer the questions.

\section{Data Processing}

The recorded data from each focus group were transcribed verbatim and translated into English. All the translations were later checked to see if they made sense. Interpretations were agreed upon by the research team. In some cases, literal translations were made and the actual words spoken by the participant were put in brackets.

\section{Data Analysis}

Analysis of the focus group transcripts was carried out manually by the study team. The analysis was done by identifying recurrent patterns and themes from both the conceptual framework and thorough reading of all transcripts. All the transcripts were read in their entirety to identify themes that re-occurred across all the focus groups. The aim of this analysis was to look for trends and patterns that reappeared within either a single focus group or among various focus groups. Other considerations related to the consistency of comments and the specificity of responses in follow up probes.

Key points, notable quotes, observations and brief summaries were written down. The analysis involved discovering emergent themes and generating reports.

\section{Results}

\section{Demographic Distribution of the Sample}

A total of 81 participants took part in 8 focus group discussions (table 1). Thirty-nine participants were recruited from Blantyre, the urban setting, and 42 participants from Chikwawa, the rural setting. All the participants were women whose children had participated or were participating in the IPTpd malaria research. The participants comprised relatively young women; over $90 \%$ of the women were aged less than 30 years. Both sites fell short of the desired male participants because those who met the eligibility criteria were only three and they did not constitute a focus group discussion. Tables 1 and 2 below show the distribution of participants, focus groups, gender, age and education by site;

Table 1: Summary of Focus Groups and distribution of participants and their gender by site

\begin{tabular}{|l|l|l|}
\hline Item & Rural & Urban \\
\hline No. of focus groups & 4 & 4 \\
\hline No. of participants & 39 & 42 \\
\hline Age range (mean) & 29.31 & 28.13 \\
\hline (Minimum) & 18 & 19 \\
\hline (Maximum) & 57 & 57 \\
\hline $\begin{array}{l}\text { Education years range } \\
\text { (mean) }\end{array}$ & 5.96 & 8.95 \\
\hline (Minimum) & 0 & 0 \\
\hline (Maximum) & 13 & 16 \\
\hline
\end{tabular}

No significant difference was observed in the age of participants from the rural and urban areas with an overall median age of 26 years.

On average, focus group participants from the urban setting were more educated than their rural counterparts with an overall median educational attainment of 9 years and 6 years respectively. Urban participants had the longest reported length of school attendance (16 years) and only $4.9 \%$ (4) of the participants had never attended school.

\section{Reasons for deciding to participate in the IPTpd Malaria Research}

Participants were asked why they had decided to take part in the IPTpd malaria research (table 2). 
Table 2: Reasons for enrolling children in the IPTpd malaria clinical research

Rural

- Wanted to get new treatment for malaria whic was very powerful/ effective

- Wanted to access better treatment than at the hospital

- Wanted to get public health education on the prevention of malaria

- Wanted to get a quick cure for malaria

- Wanted to get monetary \& material incentives such as soap, peanut butter, SOBO (orange drink), transport money, diapers, mosquito nets \& iron tablets

- Wanted to get excellent treatment and care

- Wanted to get blood transfusion for her anemic children

- Wanted to get a thorough knowledge of what the child was suffering from

- Wanted to receive special treatment normally given to research participants

Most of the reasons given above are more related to aspects of medical care found in research rather than the investigative aspect of research. In both the rural and urban settings, participants decided to take part in the IPTpd Malaria Research because they wanted their children to receive better treatment.

They also wanted to benefit from the incentives that were being given to participants which included soap, peanut butter, Orange drink, transport money, napkins, mosquito nets, basins and iron tablets.

There was also a sense of trust in health workers who asked potential participants to take part in the malaria research. Some participants felt that if they refused to participate in the research, they might not be given attention by the health workers whenever they would visit the hospital again. Thus, one participant said,

"the doctors told me that it would be of much help for me to enrol the child in the research and I had to comply because they are medical experts" (FGD 7, P6).

The participants also noted that whenever they go to the hospital for medical care, they are attended by the same health workers who also conduct research. In such a situation, they find it difficult to refuse to participate in research. On this, one participant said,

"it is the same doctors who do research. It is part of their duty. The doctors advise us to join research when they feel we will get some medical help in the research."(FGD1, P8)

Some participants also noted that they were discouraged to participate in the research at first because of the rumors that were circulating about the malaria research. They said they were rumors that the taking of blood by the researchers was linked with Satanism - that the researchers who came to conduct the research at the hospital were Satanists and their intention was to suck blood from the children and sell it for satanic activities. There was also a rumor that the blood drawn in the IPTpd Malaria Research at Chikwawa District Hospital was being taken to Blantyre for Satanic activities. Another rumor was that researchers were drawing a lot blood from the children such as a half litre of blood. Some participants also circulated rumors that the researchers took blood samples from the children to test HIV but cheated people that they were doing a malaria research so that people could rush and have their children tested for malaria. Despite the rumors, the guardians and parents agreed to enrol their children in the research because they asked the research nurses about the rumors and they were told that there was no truth to them. Those who had already participated in the research also assured their colleagues that those rumors were not true.

\section{Informed consent process}

We asked participants about the consent process before enrolling their children into the IPTpd malaria research. A majority of participants said they were asked to give their individual consent by the research team. Before giving their consent, the research staff explained the objectives of the research including the risks and benefits, as well as what was expected of the participants in the research. Those who accepted to have their children enrolled were asked to sign or thumb print the consent form and each one was given a copy of the signed consent form.

In both settings, only one participant appeared to understand that the IPTpd was a randomized controlled study. The participant stated
"They told me everything including that they were testing the effectiveness of two different drugs; quinine and another drug whose name I can't remember ... yes coartem. She also explained that the child would get the drug by chance and when I got into another room, I was given a piece of paper which I took to the doctor for medication" (FGD 8, P7).

The majority of the participants said the research nurses explained the objectives of the research including the risks and benefits. In spite of their affirmation, most of the participants did not understand the random selection.

Participants were also asked why they felt it was necessary to give their consent before enrolling their children in the research. Most of the participants said it was their right to choose to enrol their children in the research and that the researchers were respecting this right by asking for their consent. Some also said as parents (who nurse and take care of children), they had to give their permission for the children to be enrolled in the research. Thus one of the participants said
"Because it is my child and it was me who brought the child to the clinic, I had to choose whether to have my child in the study or not" (FGD 5, P7)

\section{Role of partners in the decision-making process}

Participants were asked how they came to their decisions to enrol their children in the IPT pd malaria research. A majority 
of the participants reported that they made the enrollment decisions on their own, and most of them made their decisions on the same day they were invited to enrol in the research. They informed their family members about their enrollment decision after they had given their consent about the children's participation in the IPTpd malaria research and not necessarily to seek their permission. They also said they informed their partners or relatives so that they would know where to go if a problem occurred to their children in the course of their participation in the malaria research. They also wanted them not to be surprised with their frequent visits to the hospital, and with the drugs and the transport money they used to get from the IPTpd research.

A few participants made their enrollment decisions after consulting their family members/marital partners and most of these were accompanied to the hospital by their husbands on the day they were asked to enrol their children in the research. In fact, participants felt they would consult their husbands if the husbands were with them at the hospital during the time of recruitment. For example, one of the participants said,

"...you see when a child is admitted at the hospital, you wait until he receives treatment and then you send a message bome that the child has been admitted. Having done that, the busband comes to see the child but after may be he has received several injections; but if you asked to enrol the child in a research when the busband is there, then it's a different issue. You have to consult him before you sign that the child be enrolled in the study."(FGD 1, P8).

This means participants will consult their partners if they are within easy reach. Another participant said

"If you say you will wait until you talk. to your busband, the child might not survive due to the delay in receiving treatment. So, I don't think there is any problem in informing your husband after the child has already been enrolled in the study." (FGD 7, P4).

The participants gave various reasons for consulting their marital partners before making their enrollment decisions. Some said they consulted their partners in order to seek their permission to enrol their children in the research. Others said they consulted their marital partners before making their enrollment decision because

"...it is a tradition to talk to husband and relatives before making any decision that affects both husband and wife" (FGD 2, P8 \& FGD 6, P6, P5). Table 3: Decision-making process about enrollment in the IPTpd
Malaria research

Rural

Reason for consulting/informing husbands/relatives before or after decision to participate

- Wanted to inform him about the child's participation in the study

- Because he is the head of the family

- Because marriage is between two people and children are fruits of the marriage
- Because it is a custom/ tradition to talk to husbands before making any decision that affects both of you

- Because both husband and wife are one and have to do things together, in unity

\section{Discussion}

From the responses of the participants in this study, it appears that the majority of our participants chose to participate in the IPTpd research as a way of accessing better quality medical care. Thus the inherent desire to improve one's health, sparked participants' motivation to take part in the research. In a situation of poor service delivery as is the case in Malawi, it is not surprising that medical care in a research setting is described in more favourable terms. Considering poor service delivery as is the case in Malawi, medical care in government facilities is free and the quality of care is very low, characterized by overcrowding and scarcity of basic drugs.

The findings lead us to ask the question: Can clinical research in poor- resource settings constitute an undue inducement? Is it ethically justifiable to take part in research with better quality medical care as a reason for participation within the Malawian context? If people are induced by better quality medical care, is it logical to conclude that they are "unduly" induced to participate in clinical research? According to Macklin, there are three paradigms for undue inducements in clinical research" ${ }^{12}$. In the first paradigm, she says "... inducements are undue if they prompt subjects to lie, deceive or conceal information that if known, would disqualify them as participants" - In this case, that paradigm does not apply because potential participants in the IPTpd study were approached to participate in the study after diagnostic tests showed that they met the recruitment criteria for enrollment. They were identified by the clinical officers and nurses and referred to the research clinic. In the second paradigm, Macklin states that inducements are undue if participants receive so high a payment that makes them ignore the risks in a clinical study. In our case, the participants received an equivalent of US $\$ 2$, which is a reasonable amount of money for transport reimbursement ${ }^{12}$. In our view, however, the amount was not so much that it could make them ignore the risks in the IPTpd Malaria Research. Therefore, they were not unduly induced to participate in the study. In the third paradigm, Macklin discusses the Willowbrook Hepatitis study in which third party permission was sought from parents for their retarded children to participate in the hepatitis study and also to deliberately be infected with hepatitis. The parents were motivated by the better treatment which was being given to participants and the conditions for the participants were superior to the conditions of the other children in the institution. Similarly participants in the IPTpd Malaria Research were given better treatment than the other children in the same hospital although they were not also asked to undergo a harmful procedure as part of their participation. Parents made their decisions autonomously but they were motivated by the prospect of better medical care. However, their joining the IPTpd Malaria Research on this ground is not unethical and does not constitute an undue inducement in our view. In Zeke Emmanuel's words, it "...is not an ethical worry" ${ }^{13}$. In the Malawian context, it is reasonable for one 
to make a decision to save his or her own life since failure to participate in such a research may be received as harming his or her child. African morality does not allow a person to harm oneself. It is ethical for people to choose to participate in clinical research in pursuit of better medical care.

In a scenario where the national health delivery system is overburdened and fails to adequately respond to individual's health needs, individuals are forced to take the best available option -in this case, joining clinical research. This decision was a rational one since the health system fails the people. With limited resource settings such as those prevailing in Malawi, research plays a very important role that is viewed to complement the overburdened national health delivery systems.

The findings of this study show that partners play a limited role in decision-making. Few participants made their enrollment decisions after consulting their partners or relatives. A majority of participants reported that they made the enrollment decisions on their own.

Some participants wanted to benefit from the material and monetary incentives that were being given for participation. There was also a sense of trust in health workers. Some participants felt that if they refused to participate in the research, they might not be given attention by the health workers whenever they would visit the hospital again.

\section{Recommendations}

We feel that there is need for more empirical research in African communities regarding decision making processes in order to examine some existing global assumptions on decision making in resource poor settings.

In addition, we believe it is necessary for potential participants to be adequately informed about research. They need to be (1) in a position to differentiate between clinical research and routine medical care. This is important (2) to voluntarily join a clinical research and (3) to be aware that they are participating in a clinical research at all times during participation.

Researchers also need to adopt innovative methods of teaching communities and individuals about research. Before initiating any clinical research in communities, researchers should sensitize the public regarding the research project. This would also encourage people decide to participate in research, based on adequate information which is passed in a way that makes it easy to understand. As long as people make their decisions based on adequate information; and participate in order to avoid the challenges that the national health delivery presents, such decisions are rational and ethical.

Finally, researchers should feel responsible for the needs of the communities where they conduct research by, for example, supporting clinics that are open to all members of the communities and not restricted to the patients or even disease under study. We believe that clinical research presents one route to accessing health care.

\section{Conclusion}

The results of this study show that a majority of participants decided to participate in the IPTpd Malaria Research because they wanted to get better treatment that was being given to participants. Some participants wanted to benefit from the material and monetary incentives that were being given for participation, for example, soap, peanut butter, Orange drink, transport money, napkins, mosquito nets, basins and iron tablets. There was also a sense of trust in health workers who asked the potential participants to take part in the malaria research. However, some participants felt that if they refused to participate in the research, they might not be given attention by the health workers whenever they would visit the hospital again.

The findings also show that partners play a very small role in decision-making. According to the findings, a few of the participants made their enrollment decisions after consulting their partners or relatives and most of these were accompanied by their husbands to the hospital on the day they were requested to enrol their children in the research. In fact, a majority of participants reported that they made the enrollment decisions on their own and they informed other family members about their enrollment decisions only after they had given their consent in order to let their partners know about their children's participation in the IPTpd Malaria Research and not necessarily to seek their permission.

The results also show no association between the level of education of participants and their decisions to inform or not to inform their partners or relatives about their participation in the IPTpd Malaria Research.

Finally, the results did not show any association between participants' locations and their decisions to inform or not inform their families about their participation in the IPTpd Malaria Research.

\section{Acknowledgements}

This research project was supported by the Johns Hopkins -Fogarty African Bioethics Training Program with funding from the National Institutes of Health Fogarty International Center, the National Institute of Environmental Health Sciences and the National Heart Lung and Blood Institute (NIH Research Grant \# D43 TW001604).

We would like to thank; Nick Juul, Jennifer Lohse and Joseph Ali at the Johns Hopkins University, USA; Thressa Likomwa and Mr. Wiskesi in Malawi; and The Management, staff and research participants from: The IPTPD Malaria Clinical Trial at Queen Elizabeth Central Hospital in Blantyre, and at Chikwawa District Hospital in Chikwawa.

\section{References}

1. Ijsselmuiden C B, Faden R. Research and informed consent in Africa; Another look. N Engl J Med 1992; 326:830 - 4.

2. Creed-Kanashiro H, Ore B, Scurrah M, Gil A, Penny M. Conducting Research in Developing Countries: Experiences of the Informed Consent Process from Community studies in Peru. The American Society for Nutritional Sciences J. Nutr. 2005: 135:925-928. http:// www.nutrition.org/cgi/content/full/135/4/925

3. Tindana P, Kass N, Akweongo P. The Informed Consent Process in a Rural African Setting: A Case Study of the Kassena-Nankana District of Northern Ghana. IRB: Ethics \& Human Research. Hastings Center. 2006: Vol. 28, No. 3

4. Sewankambo, N. Report on the National Consensus Conference in Uganda. 1997

5. Guidelines for the Conduct of Health Research Involving Human Subjects in Uganda, 1997

6. Pace C, Talisuna A, Wendler D, Emanuel E, Grady C. Quality of 
parental consent in a Ugandan Malaria Study. American Journal of Public Health; 2005: Vol. 95. No. 7. $1184-1189$

7. Bhan A, Majd M, Adejumo A. Informed Consent in International Research: Perspectives from India, Iran and Nigeria. MUMJ Medical Ethics; Vol. 3. No. 1. 2006: pp. 36 - 41

8. Caballero B. Ethical issues for collaborative research in developing countries. American Journal of Clinical Nutrition. 2002:Vol. 76, No. 4,
717 - 720. http://www.ajcn.org/cgi/content/full/76/4/717

9. Hebert PC, Doing Right: A Practical guide to ethics for medical trainees and Physicians. New York. Oxford University Press: 1996

10. National Bioethics Advisory Commission (NBAC) report. Ethical and Policy Issues in International Research. 2005

11. Phase II Report of the Wellcome Trust Bioethics Research Project, 2007

\title{
Call for Articles for a Special Issue on Cancer and Palliative Care
}

Malawi Medical Journal Vol 20 Issue 4, December 2008 will be a special issue on Cancer and Palliative care. Authors are invited to submit articles via the online submission system mc.manuscriptcentral.com/mmj or emailmmj@medcol.mw

\author{
You are welcome to submit the following \\ Case reports \\ Letters to the Editor \\ Poems \\ Photos \\ Interviews \\ Profiles on organization \\ Short reports \\ Reviews \\ Original research articles \\ Editorial Opinions
}

Articles should be submitted no later than 30th September

For more information please email mmj@medcol.mw or call 01878524 